\title{
Prosthodontic Rehabilitation of a Patient with Unilateral Facial Paralysis: A Rare Clinical Report
}

Preeti Sagar $^{1}$, Manesh Lahori ${ }^{2}$

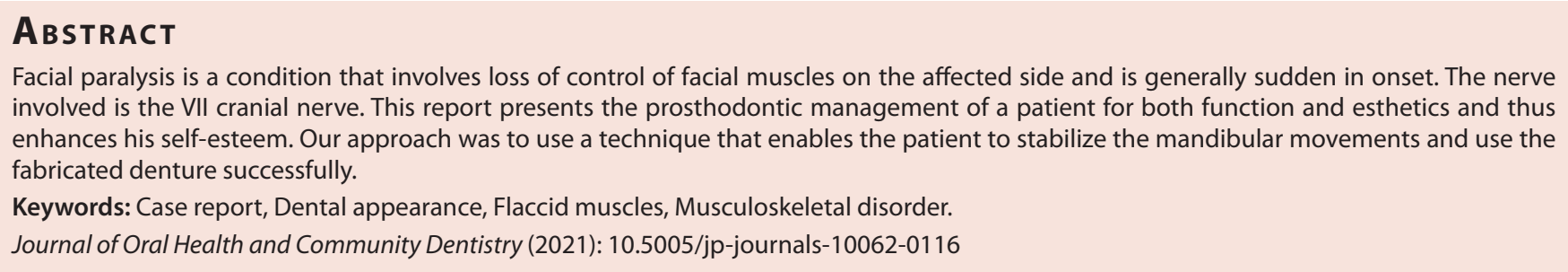

\section{BACKGROUND}

Facial paralysis is a condition that involves loss of control of facial muscles on the affected side and is generally sudden in onset. The nerve involved is the VII cranial nerve. The commonly involved etiological factors are immune or viral diseases (herpes zoster oticus), trauma (iatrogenic, accidental), ischemia of the nerve (neoplasms), or idiopathic (Bell's palsy). ${ }^{1}$ Based on the site of the neurons affected they are classified as upper motor neuron (UMN) and lower motor neuron (LMN) paralysis. ${ }^{2}$

The clinical findings associated are inability to smile and close eyes on the affected side, reduced taste sensation, spasms of facial muscles, and asymmetry of face. Tests such as the blink test, facial expressions, stapedial reflex checking, Schirmer test, and taste testing in the anterior two-thirds of the tongue support the diagnosis. $^{1}$

\section{Case Description}

A 60-year-old male patient reported to the Department of Prosthodontics with partially missing teeth. The patient gave a history of facial paralysis on the left side of his face 8 months back, after which he experienced facial asymmetry, mouth deviation, drooping of food from the left corner of the mouth, absence of wrinkles on the forehead, and slurred speech (Fig. 1).

Extraoral examination revealed asymmetry of the left side of his face when he tried to smile or close his eyes with a maximal effort. House-Brackmann grade IV-moderately severe dysfunctionmay be applied to the paralysis. ${ }^{3}$

On intraoral examination, a well-rounded, firm completely edentulous maxillary ridge and partially edentulous mandibular ridge was noticed. The remaining mandibular teeth were attrited and periodontally weak. Oral prophylaxis was carried out to reduce periodontal risk to the remaining teeth.

Our treatment plan was a modified complete denture with respect to the maxillary arch and a plate removable partial denture (RPD) for the lower arch having monoplane teeth.

Primary impressions were made for the upper and lower edentulous arches using irreversible hydrocolloid. A diagnostic cast was obtained. Custom trays were constructed for upper and lower arches using autopolymerizing polymethyl methacrylate resin.
1,2 Department of Prosthodontics, KD Dental College and Hospital, Mathura, Uttar Pradesh, India

Corresponding Author: Preeti Sagar, Department of Prosthodontics, KD Dental College and Hospital, Mathura, Uttar Pradesh, India, Phone: +918867637861, e-mail: pssagarpreeti@gmail.com

How to cite this article: Sagar P, Lahori M. Prosthodontic Rehabilitation of a Patient with Unilateral Facial Paralysis: A Rare Clinical Report. J Oral Health Comm Dent 2021;15(3):144-145.

Source of support: Nil

Conflict of interest: None

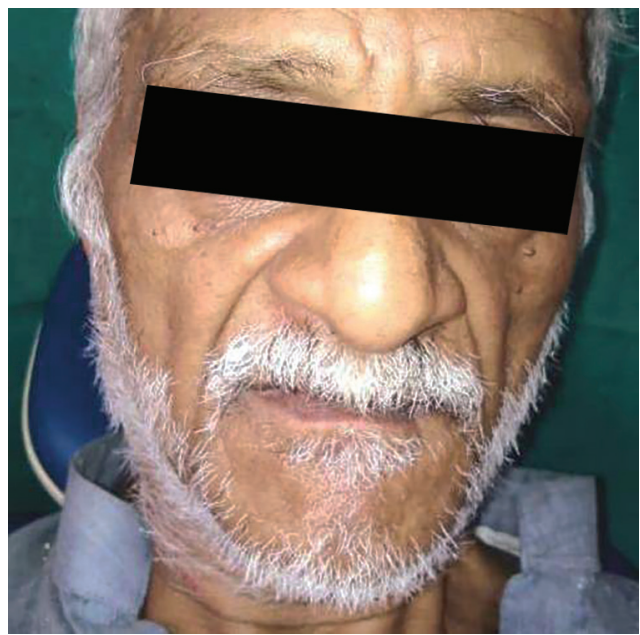

Fig. 1: Preoperative photograph of the patient

Border molding was done, and final impressions were obtained. The borders of the affected side were made thick to add adequate buccal sulcus support (Fig. 2). The preserved lower teeth were used to determine the occlusal vertical dimension.

To replicate the symmetry of his face and preserve esthetics, the anterior teeth were set according to the shifted midline. The monoplane posterior teeth were selected as they help in easier closure for uncoordinated movements, prevent lateral stresses, and 


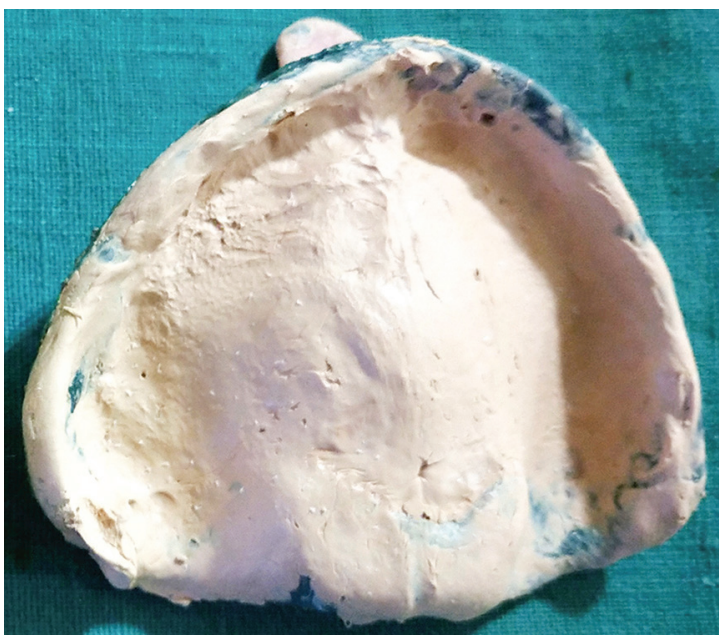

Fig. 2: Final edentulous impression with thick buccal sulcus support on the left side

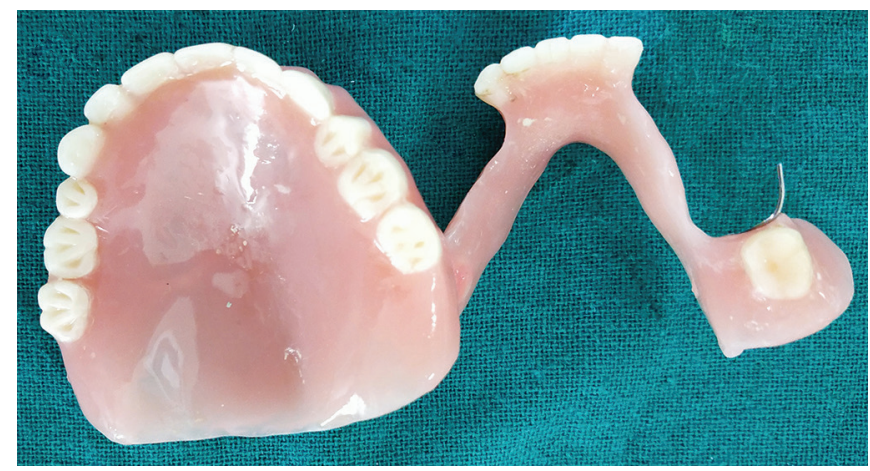

Fig. 3: Finished prosthesis with monoplane teeth

reduce the damage to the tissues that support the denture. After try-in, wax up and carving were carried out. Heat-cured PMMA resin was used to make the upper complete denture and the lower partial denture. The dentures were finished and polished (Fig. 3) and insertion was carried out (Fig. 4).

The patient was given proper postop instructions for use and maintenance. Oral hygiene was emphasized to avoid food entrapment between the dentures and the cheek as well as to maintain oral tissues in healthy condition.

Patient follow-up was scheduled at an interval of 1, 3, and 6 months. The patient had satisfactory response as he can chew better now and had improved appearance (Fig. 5).

\section{Discussion}

The main aim of prosthetic treatment should be to support the weakened muscles, restore facial appearance, and maintain tonicity of the surrounding tissues. Our approach of prosthetic rehabilitation with respect to the abnormal condition was executed methodically. However, the treatment choice does not end here, and the patient should be referred to an ophthalmologist for the treatment of the paralyzed eyelid to enhance appearance, function, and selfconfidence in the patient.

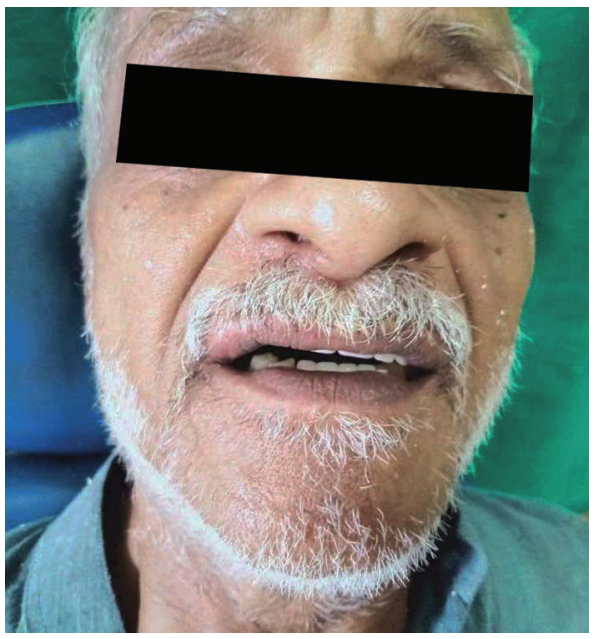

Fig. 4: Postoperative photograph of the patient with upper complete denture and lower partial RPD
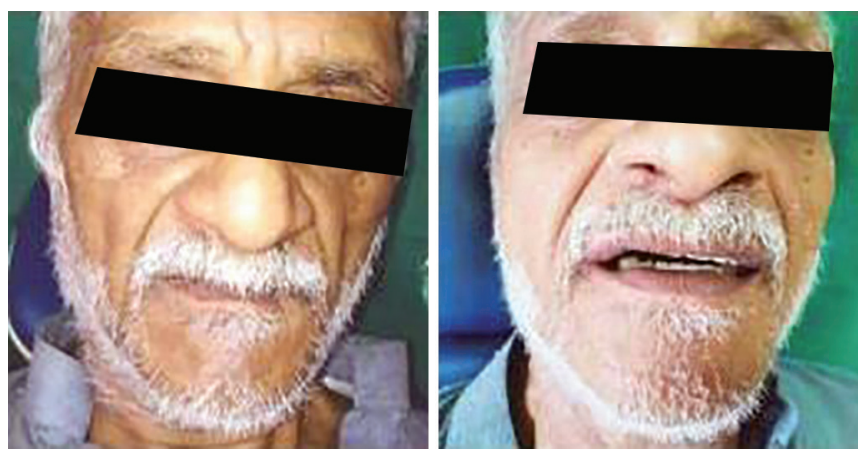

Fig. 5: Pre- and postoperative photographs of the patient

\section{Conclusion}

A corrective surgical procedure of the affected nerve would be the ultimate cure for any unrecovered facial paralysis. However, in complicated cases where surgery is not a choice, oral prosthesis plays a vital role in the patient's well-being.

\section{Clinical Significance}

The patient had satisfactory response as he can chew better now and had improved appearance and speech. Monoplane teeth selected for the rehabilitation of the facial paralyzed patient were found to be very effective in minimizing damage to the tissues supporting the denture and preventing lateral stresses, and also helped in easier closure for uncoordinated mandibular movements.

\section{References}

1. Pandey S, Datta K. Prosthodontic management of a completely edentulous patient with unilateral facial paralysis. J Indian Prosthodont Soc 2007;7(4)211-213. DOI: 10.4103/0972-4052.41076.

2. Chaurasia BD. In: Chaurasia BD, editor. Human anatomy. 2nd ed. p. 41-113.

3. House JW, Brackmann DE. Facial nerve grading system. Otolaryngol Head Neck Surg 1985;93:146-147. DOI: 10.1177/019459988509300202. 